\title{
NIH Grant Watch
}

\section{Real-Time PCR}

Some 250 grants awarded by the U.S. National Institutes of Health over the past year have featured applications of real-time PCR. Here, we summarize a selection of some of the largest of these grants, with concentrations on cancer and infectious diseases.

DNA Methylation and Chromatin Modifications: Mechanisms and Applications in Cancer Samson T. Jacob

(5P01CA101956-02, National Cancer Institute, 08/01/07)

Ohio State University, Columbus, $\mathrm{OH}$

$\$ 2,215,284$

Goal: To advance basic understanding of epigenetic regulation of gene expression (and its reversal) in cancer cells and rapidly translate understanding of molecular mechanisms into clinical practice.

Molecular-Nutrient Interactions in Intestinal Cancer

(5U54CA100926-05, National Cancer Institute, 08/16/07)

Leonard $\mathrm{H}$. Augenlicht

Montefiore Medical Center, Bronx, NY

$\$ 1,987,302$

Goal: To understand how a Western-style diet interacts with genetic factors to increase probability of tumor formation in the intestinal tract.

\section{Center for Experimental Infectious Disease Research}

(5P20RR020159-04, National Center for Research Resources, 06/15/07)

Konstantin G. Kousoulas

Louisiana State University A\&M College, Baton Rouge, LA

$\$ 1,924,234$

Goal: Five integrated research projects investigating molecular and immunological aspects of viral infections.

HT-Film-Array: A System to Assess Respiratory Viruses with Emphasis on Influenza (1U01AI074419-01, National Institute of Allergy and Infectious Diseases, 08/07/07)

Steven F. Dobrowolski

Idaho Technology, Salt Lake City, UT

$\$ 1,793,553$

Goal: To develop a dense format to concurrently assay for a panel of respiratory viruses, including influenza, and to characterize influenza $\mathrm{H}$ and $\mathrm{N}$ genes to differentiate strains.

\section{Biological Breast Cancer Classification by qRT-PCR}

(5U01CA114722-03, National Cancer Institute, 06/01/07)

Matthew J. Ellis

Washington University, St. Louis, MO

$\$ 1,549,377$

Goal: To identify prognostic classification of breast tumors via RNA expression profiling.

\section{Sample Processing Cartridges for Rapid PCR TB Detection}

(5R42AI052523-03, National Institute of Allergy and Infectious Diseases, 08/14/07)

David Alland

Cepheid, Sunnyvale, CA

$\$ 1,343,564$

Goal: To integrate sample processing and real-time PCR diagnosis into a single, hands-free process.

Detection of Select Agents in Single-Well Assays

(5U01AI056689-05, National Institute of Allergy and Infectious Diseases, 06/18/07)

David Alland

University of Medicine and Dentistry of New Jersey, New Jersey Medical School, Newark, NJ $\$ 873,498$

Goal: To develop rapid, simple, robust, and specific assays for pathogenic bacteria (particularly potential bioterror agents) via novel approaches to real-time PCR that expand the number of different pathogens detectable in a single assay well.

Sensor Technology for Rapid Microbial Identification and Susceptibility Testing

(1U01AI075565-01, National Institute of Allergy and Infectious Diseases, 06/25/07)

David A. Haake

Brentwood Biomedical Research Institute, Los Angeles, CA

$\$ 680,764$

Goal: To develop all of the essential technological components needed to produce a biosensor for rapid high-throughput bacterial pathogen identification and antibiotic susceptibility determination, via an electrochemical sensor for rapid genotypical identification. 